\title{
The Ambient Horn: Designing a novel audio- based learning experience
}

\author{
Cliff RAndell*, SARa Price**, Yvonne Rogers***, ERIC Harris**, Geraldine \\ FITZPATRICK** \\ * Department of Computer Science, University of Bristol, Bristol, BS8 1UB, UK \\ cliff@compsci.bristol.ac.uk \\ Fax: +44 1179545208 , Tel: +44 1179545104 \\ **School of Informatics, University of Sussex, Brighton, BN1 9QH, UK \\ ***School of Informatics, Indiana University, USA
}

\begin{abstract}
The Ambient Horn is a novel handheld device designed to support children learning about habitat distributions and interdependencies in an outdoor woodland environment. The horn was designed to emit non-speech audio sounds representing ecological processes. Both symbolic and arbitrary mappings were used to represent the processes. The sounds are triggered in response to the children's location in certain parts of the woodland. A main objective was to provoke children into interpreting and reflecting upon the significance of the sounds in the context in which they occur. Our study of the horn being used showed the sounds to be provocative, generating much discussion about what they signified in relation to what the children saw in the woodland. In addition, the children appropriated the horn in creative ways, trying to 'scoop' up new sounds as they walked in different parts of the woodland.
\end{abstract}

Keywords: augmented reality, mobile learning, pervasive computing, audio-based learning

\section{Introduction}

Mixed reality environments, where the digital world is combined in some way with the physical world [7], provide opportunities to augment our experiences of the everyday world. Sensor-based devices can be triggered to deliver digital information through various human bodily movements in a variety of contexts - quite unlike those we are used to with conventional computer interfaces (e.g., VR, multimedia). One area where there is much potential for developing novel forms of experience is learning. In particular, active forms of learning can be encouraged, provoking 
children to stop, think and wonder, by combining the familiar with the unfamiliar and the expected with the unexpected [e.g. 1, 9, 14, 15, 19, 20, 21, 23].

Within this vein, the Ambient Wood was designed as a learning experience for children to discover, explore and reflect upon the complex processes that exist in a woodland ecology [24]. A woodland area was digitally enhanced through transforming it into a mixed reality environment. A number of digital representations of underlying physical processes were engineered to appear at contextually relevant times. These included images, sounds and videos conveying processes like photosynthesis and pollination. One of the main aims of developing this form of digital/physical augmentation is to provide abstractions relating to the 'hidden' processes of the woodland's ecology, not normally available to see, hear or have access to when walking around outdoors. Importantly, in so doing, our objective was not to bombard children with information while exploring the woodland, but to provide, at certain times, access to relevant knowledge that would enable children to reflect upon the important factors and processes that underlie the woodland ecology.

A variety of devices were used to deliver and gain access to the digital augmentations, including PDAs, wireless speakers, a probing device for collecting readings and a periscope device. The design and evaluation of these are reported elsewhere $[24,26]$. In this paper, we describe the design of a device called the Ambient Horn, a novel mobile sound delivery system that was developed for our latest version of the Ambient Wood learning experience. The Ambient Horn plays abstract sounds at various locations in a woodland, intended to represent ecological processes that are normally inaudible and invisible, such as plant respiration, root uptake and bee pollination.

The reason for selecting these kinds of higher-level abstractions is that children tend not to think about them when engaged in their explorations of the 'here and now' of a woodland. We wanted to draw their attention at relevant times to what goes on behind the scenes and enable them to relate this to what they can see and hear with their own eyes and ears. In particular, we wanted them to notice various features of a 
woodland that would enable them to reflect on how these related to the abstract processes that lay behind them. In so doing, we needed to ensure that the actions involved in collecting and listening to the ambient sounds would not detract from the children's interactions with the physical world. A key concern, therefore, was to determine whether the ongoing activity of exploring the woodland could be enhanced with unexpected augmented sounds. A further aim was to investigate the kinds of sounds that would be most effective at provoking children into reflecting and interpreting.

\section{Relevant background}

Speech is fundamental to learning. Much educational research has focused on how best to support learning using different models of dialogue. Of interest here is how the use of non-speech audio can enhance learning. Many different kinds of abstract sounds can be created, based around the parameters of pitch, rhythm, intensity and timbre. These can be used in various ways and contexts: as an indicator that provides shift of emphasis in speech; as an interpreter of representations; as a 'tap on the shoulder' to gain attention to point out something of interest; and as a way of embellishing other experiences of an event that is happening or about to happen. Within HCI, non-speech audio has been used to augment conventional output devices such as a visual display, providing the user with audio cues for specific events. Such sounds are sometimes known as 'earcons' - "non-verbal audio messages that are used in the computer-user interface to provide information to the user about some computer object, operation or interaction" [3] and have been found to be particularly good at capturing a user's attention whilst they are performing other tasks [5, 18].

Within learning contexts, non-speech audio has been used to make clearer and simpler certain kinds of complex information. For example, sound has been found to mediate understanding of large amounts of abstract data in complex systems, by marking differences in the data variables $[4,6]$. A particular form of non-speech audio that is used is 'sonification', defined by Kaper et al. [16] as the "faithful rendition of data into sounds", where abstract sound variables are parameterised by modifying their frequency, amplitude and duration to map data, often happening in 
real time. Sounds used for sonification are normally composed of synthesized tones and have been shown to be effective at helping undergraduate students learn about molecular properties when used in combination with 3D visualizations [11].

As when designing any kind of representation - be it visual, auditory or other - an important concern is what form to use and how this maps onto the underlying referent. The kinds of mapping used can be arbitrary, symbolic or direct [22]. An example of an arbitrary mapping is of a 'tong' sound to represent happiness. There is no relationship between the two. Equally, a 'ting' could have been used. An example of a symbolic mapping is the sound of glass smashing to represent fragility. Here the sound conveys an underlying referent that is at a higher level of abstraction than the sound itself. An example of a direct mapping is of a clapping sound used to represent clapping. The sound resembles the action and can be readily interpreted in the way it is meant to be. For some kinds of referents it is relatively easy to design sounds that have direct mappings. For other kinds of referent, however, especially those that are abstract (e.g. radiation), it is difficult to design representations that have direct mappings. In these instances arbitrary representations have to be used (although over time some of them become universally understood such as the symbol of overlapping broken circles to represent radiation).

Gaver's [12] auditory icons, developed as part of the SonicFinder (an experimental operating system for the Mac interface) mainly used direct mappings. A set of auditory icons was created to represent actions performed on various interface objects. They were intended to semantically map onto naturally occurring sounds that would be heard if the same actions were performed with counterpart physical objects. For example, the sound of 'thunk' was used to represent dropping a file into a folder and 'crash' for deleting an object when dropped into the trashcan. In this interface context, the non-speech sounds were used to emulate, in an exaggerated form, physical actions, and in so doing providing feedback to alert the user's attention to various interface events. Other research into non-speech audio has used more arbitrary mappings, for example, the use of various musical sounds to help users debug when programming [10]. 
Here, we are interested in whether a variety of arbitrary and symbolic sounds, representing abstract and invisible ecological processes, can promote reflection in children. The sounds are designed to be suggestive rather than literal, using arbitrary and symbolic mappings. Our aim was to promote curiosity and discussion among the children as to what the sounds signified, and how they related to what they could see and hear around them.

\section{Design of the audio based learning experience}

In our first design of the Ambient Wood learning experience [24] a number of digital sounds were pervasively presented to the children. Whenever they moved to a location in the woodland where an RF location beacon, or 'pinger', was hidden, their bodily presence triggered a sound that was played through nearby wireless loudspeakers, also hidden. This design was aimed at giving a richer experiential texture to the learning experience where digital sounds of woodland organisms were added to the natural environment. These included: animal sounds (e.g. bird singing, caterpillar eating, butterfly drinking nectar) and plant sounds (e.g. thistle dying, grass rustling, leaves decomposing). One goal of using this pervasive technique was to provide an element of surprise. If the children walked past a hidden beacon, a particular sound would be triggered, but the children would be unaware when this might happen and what caused it. This technique was intended to stop the children in their tracks and figure out what the sound signified and why it had happened. Moreover, we wanted the sound to draw the children's attention to aspects of the habitat they might not otherwise notice, providing relevant contextual information that they could integrate with their experience. For example, if the children walked past a certain bush that attracted butterflies a sound of a butterfly drinking nectar would be played. The aim was for children to reflect upon this unusual sound and work out the interdependency between the flowering of the bush and the butterflies feeding upon it.

However, preliminary findings of the first Ambient Wood trials showed that the ambient sounds provided in this manner appeared to "fit" into the setting so well that 
they were hardly ever noticed by the children. Even when a facilitator (who stayed close by the children during their explorations) drew their attention to the sounds after they had been played, the children would look somewhat puzzled, having not heard them. This raises the question of whether our original design of ambient sounds was in fact too ambient that they were simply not heard among the 'noise' of the other naturally occurring sounds in the wood. Most of the time, the children were simply too engrossed in other things to even notice a sound [24]. Kilander and Lönnqvist [17] consider the "subtle difference between the anticipated and the perceived" suggesting the importance of understanding the meaning of the effect (in this case, sound). If sound is perceived as meaningful then the effect is to attract attention, whereas anticipated sound merely 'fades into the background'.

\subsection{The design of the Ambient Horn}

To overcome the 'noise' problem of the children missing the pervasively delivered sounds we decided in a subsequent design of Ambient Wood to give the children control over the playing of the sounds, where they have to physically interact with a handheld device in order to listen to them. The Ambient Horn was designed to still maintain the effect of surprise by the apparent serendipitous triggering of digital information (still using location pingers), but also enabling the children to choose exactly when to play the sounds. A simple interface was designed, using indicator lights to alert the children to the presence of a sound and a button to enable them to play (and replay) the sound. Thus the sounds were still accessible in a contextually relevant part of the wood, but the horn enabled the sounds to be kept 'on hold' until the children themselves were ready to listen to them. This way, the children could remain engrossed in their ongoing activities until one of them noticed that a sound had been triggered ready to be played.

The original design concept for the Ambient Horn was to acquire real or plastic horns in accordance with nature and the woodland environment (see Figure 1). However, horns such as these were not large enough to house the envisaged technology. In earlier trials we observed children's fascination for novel technology and believed that a technically overt design would be more engaging. 


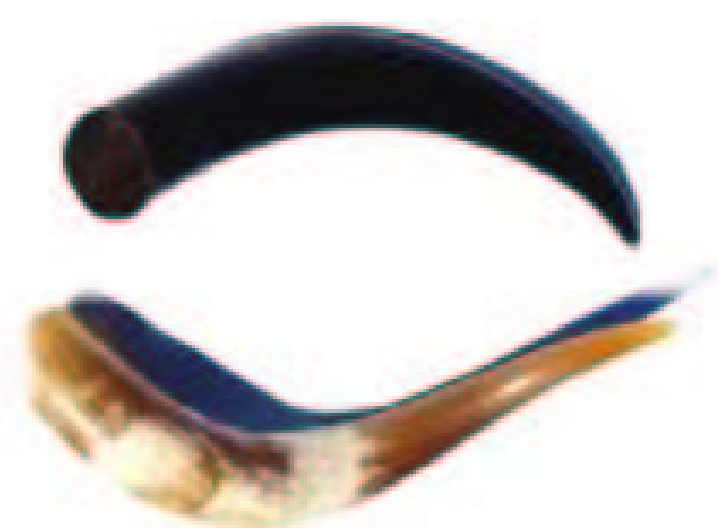

Figure 1. Original horns used as inspiration for the design concept

In our previous work with the Periscope [26] we explored the appeal of a hybrid design combining high technology with forms which echo elements of a natural, organic environment. The idea of creating hybrid forms which directly reflect the environment, the intended or perceived use and the user, no matter how abstractly, can lead us to consider how to design more aesthetic, creative and unusual appliances, but which at the same time are still effective and useful for the tasks they have been designed to support. The first prototype we built was the Box-Horn ('box+horn'), attempting both to echo the organic shape of the natural horn, and at the same time provide an interesting technical object. An MP3 player was mounted inside the white box, two red Light Emitting Diodes (LEDs) flash when a location ping is received by the horn and the sound is played through the metal horn by operating a push button (figure 2).

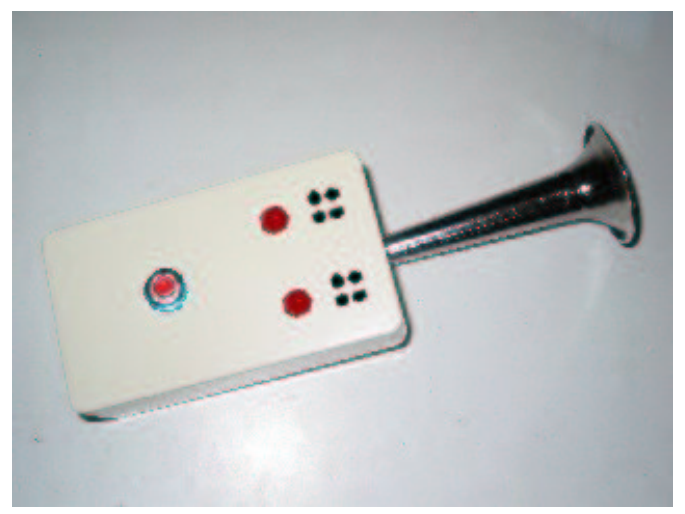

Figure 2. The Box Horn prototype 
The Box Horn prototype was found to be unsatisfactory because of its size (being too large for small children to operate conveniently) and limited control functions. Anything beyond pushing the play button required the case to be opened. To overcome these drawbacks, the Techno-Horn was developed (figure 3). Here, the MP3 player was positioned outside the box providing a smaller and much more visible 'techy' solution with the possibility that the children could operate the player themselves as well as having sounds automatically cued. The horn itself was again used as a speaker housing.

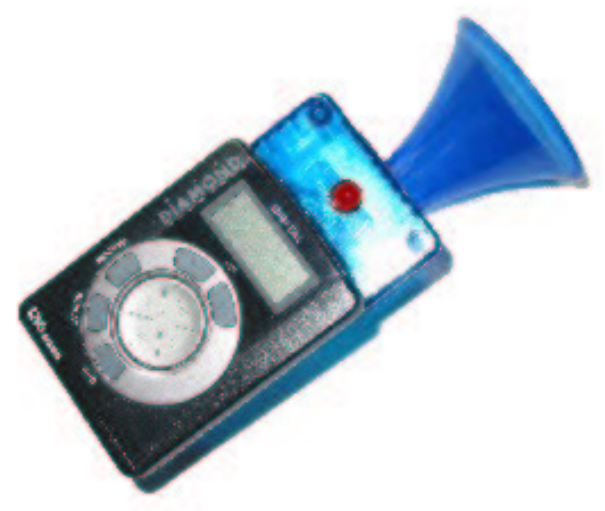

Figure 3. The Techno Horn prototype

However, trials with this prototype showed the device to be bulky and awkward for the children aged 10-11 years to operate while moving around. The remote cueing of the MP3 player required 1 second per track, e.g. 8 seconds to cue track 8 , providing delays, which reduced the spontaneity of the device's performance. This design also required children to learn the additional functions of the MP3 player.

To overcome these limitations the MP3 player was replaced with a customised sound chip. This reduced the overall size of the device, and enabled a bespoke interface to be incorporated instead of using the proprietary design. Although the specification of the sound chip is more limiting than a MP3 player (with only 60 seconds of monophonic audio available) the speed of response is almost instant, and by using looping techniques we could provide an equivalent range of sounds to those previously played by the MP3 player. 
The final design of the Ambient Horn was intended to be simple to operate, compact to hold easily in one hand, and robust for outdoor mobile use. The horn emits a distinct 'honk' when a ping is received, drawing attention to the presence of a sound. In addition, two LEDs, integrated into the horn, continue to flash until the sound is played. If the children miss the audio cue or wish to continue with what they are doing the LED lights act as a reminder that a sound is waiting. A push button triggers the sound to be played. In accordance with the design of the activity, a rotary switch was built onto the device to access two alternate sets of sounds (one focusing on plant processes and the other on animal activities). The horn was also fitted with a drawstring so that it could be worn on the body as a pendant, freeing up the child's hands to use other devices (e.g. a walkie-talkie or PDA). When worn, like this the LED lights are visible for the other child to notice and initiate collaboration in its use.

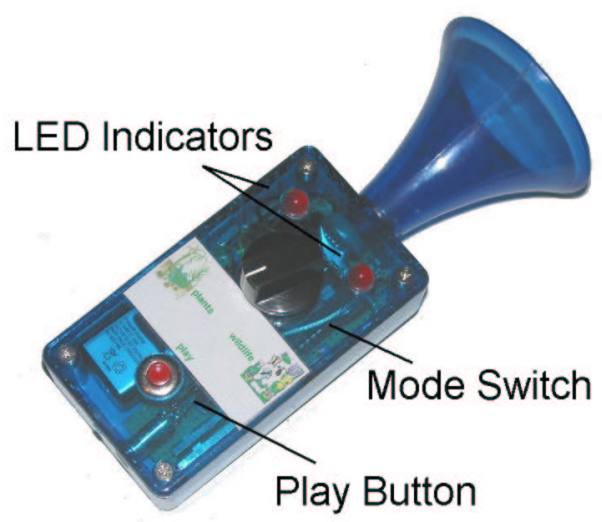

Figure 4. The final design of the Ambient Horn

To enable the Horn to trigger the sounds in context the receiver was engineered to detect proximity to location pingers. The set up was designed such that when the children moved out of a designated pinger area, but then came back into the same area, they received again the sound associated with that pinger.

The location pingers had a range of 10 meters and were deployed at various points of interest in the wood such as in thistle patches and reed beds. The Ambient Horn itself generated a ping signal every time it was used to enable a record to be kept of its 
usage by creating notifications of events to a network server as they happened. These pings were processed via a bodyworn local receiver attached to a wirelessly networked PDA. The wireless network provided coverage for the total area of the wood explored by the children. This was achieved using three access points running on the IEEE 802.11b standard and supplied a backbone to which the Horn and the other Ambient Wood devices could connect.

\subsection{The design of the sounds}

Similar to the design rationale for the first Ambient Wood trials, our aim was to design a set of sounds to be played with the Ambient Horn that could facilitate children's reflection and discussion as to their meaning and significance. Abstract animations have been found to promote reflection, creativity and imagination in children [19]. Likewise, we supposed that abstract sounds could provoke reflection, by requiring the children to interpret the sounds based on what they know and what they see around them. To this end, we chose a variety of sounds to represent a range of ecological processes that take place in the woodland; processes that are invisible to the naked eye, but are contextually relevant for understanding habitat interdependencies.

We primarily used two kinds of mappings, arbitrary and symbolic. An example of an arbitrary mapping was the use of an energy kind of sound (like a light sabre) to represent photosynthesis. Here, our aim was to provoke children into understanding the factors involved in photosynthesis, identifying the different aspects that were visible to them in the woodland. An example of a symbolic mapping was the use of a chomping sound to represent animal eating behaviour, at a higher level of abstraction than the sound itself. Children could receive eight sounds in total, four were plantbased and four animal-based. The type of sound, mapping used and underlying process being represented is shown in Table 1. As can be seen three of the plantbased mappings were arbitrary while three animal-based were symbolic. The plant processes, being more abstract, could only be represented by arbitrary sounds, whereas the animal processes lend themselves more to symbolic mappings. 
We also deliberately limited the number of sounds that could be heard via the horn as we did not want 'sound collection' to become the dominant activity of the learning experience in the wood.

\begin{tabular}{|llll|}
\hline \multirow{7}{*}{ Plant-based } & Process & Sound & Mapping \\
& Photosynthesis & Light sabre sound & Arbitrary \\
& Respiration & Fireworks & Arbitrary \\
& Decomposition & Paper rustling & Arbitrary \\
& Root uptake & Water slurping & Symbolic \\
Animal-based & Bee pollinating & Rasping & Arbitrary \\
& Squirrel eating & Crunching & Symbolic \\
& Caterpillar eating & Chomping & Symbolic \\
& Animal moving & Fast tapping & Symbolic \\
\hline
\end{tabular}

Table 1. Mappings used between the ecological process and sound used to represent it

\section{Experiences with the Ambient Horn and sounds}

The Ambient Wood was intended to promote a range of learning experiences, based around the process of scientific enquiry. It involved pairs of children collecting, probing, exploring, hypothesizing and evaluating and comparing their findings. Details of the learning outcomes are reported elsewhere [24]. Of interest, here, is how the children used the horn in their exploration of the woodland. To promote further reflection on what they had just heard the children were required to report their findings to a remote facilitator, using a walkie-talkie. This was intended to encourage them to articulate their discoveries at another level of description, forcing them to be explicitly aware of their own and one another's understanding. Once the sound has been discussed with the remote facilitator further information was sent by the facilitator (e.g. an image) onto a PDA, they were also carrying with them.

\subsection{Use of the Ambient Horn}

Overall, the Ambient Horn proved to be successful for augmenting learning; it acted as a tool that promoted reflection, interpretation and further exploratory activity in the woodland. Twelve pairs of children, aged between 11-12 years used it.. They found it 
easy to use, very engaging, and showed great interest and eagerness to listen to the sounds. Several children expressed great enjoyment at using it"

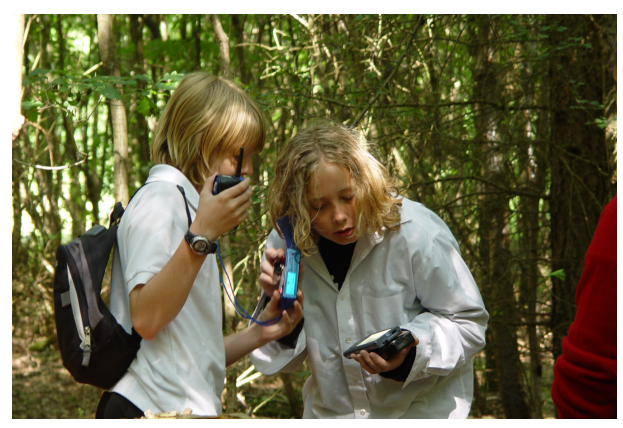

Figure 5. Using the Ambient Horn to listen to a sound

The children intuitively interacted with the horn itself in a variety of ways. Some pairs of children held it to their ear to listen to the sound as might be expected. In these instances they took it in turn to listen to the sound, with sometimes one child holding it for the other child to hear. Other pairs chose not to hold the horn to their ear, but held it in front of them, enabling both children to listen at the same time. The design allowed children to collaboratively engage with the device and encouraged good sharing practice (figure 5). The facility to hang it around the neck left their hands free for other devices, such as PDA and walkie-talkies, and its shape and size enabled easy grasping in one hand, allowing easy transferring between children.

The Ambient Horn was successful in attracting the children's attention to the sounds. When the horn 'beeped' the students often stopped immediately and listened to the sound that was played. However, if a sound arrived when the children were already engaged in other activities in the wood (e.g., probing the environment for a light reading) they often continued with what they doing before listening to the sound. There were also times when the child carrying the horn was engrossed in another activity (e.g., talking with the remote facilitator on the walkie-talkie), and the other child having noticed the LEDs flashing, was the one to initiate interaction with the horn. Our findings suggest, therefore, that marking the arrival of a sound with a 'beep', 'storing' sounds, and using LEDs in this way, enabled the children to manage their interactions with the digital information. 


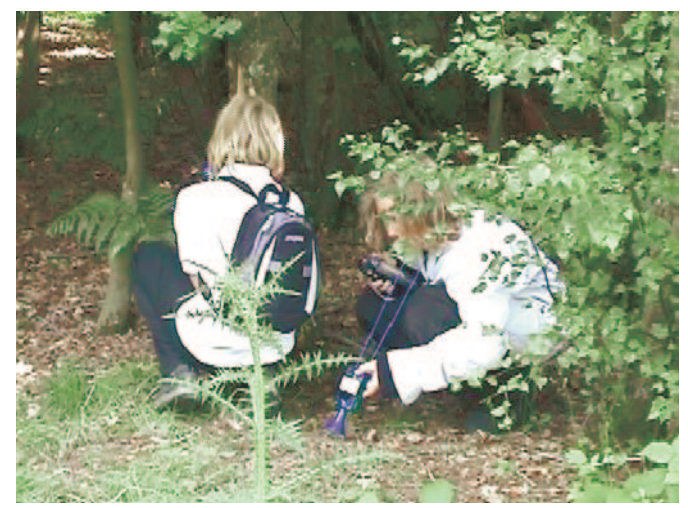

Figure 6. A pair of children experimenting with the Ambient Horn

Although the Ambient Horn was designed to receive sounds triggered by pingers according to location, many of the children used the horn in unanticipated ways to interact with their environment. Some perceived it as an instrument that could collect sounds. Their actions suggest that they appropriated the tool as a collecting device, choosing what kinds of things to hear, for example, taking it to different plants, thus, naturally associating a sound with an item or object. Several children also made scooping actions with it through the air, as if to catch a sound, exploring different places where they might get sounds. One child interestingly used the verb to 'take' when talking with her peer "shall we take another sound?" Another pair of children used it to try to find out more about the woodland. After hearing the photosynthesis sound they held the Ambient Horn over a leaf in the sunshine as if to see whether phototsynthesis was taking place.

\subsection{Reflection and interpretation of the sounds}

Our observations of the children discussing the sounds with each other and the remote facilitator showed evidence of attempts to relate what they had heard with what they saw around them and the implications of this in relation to the ecological processes. The most effective sounds at triggering this kind of reflection among the children were those with symbolic mappings. For example, children explored the relationship between the habitat characteristics and the sound they received. One pair of children interpreted the root uptake sound (a water- related sound) as rain when in a clearing part of the woodland. They reasoned that rain was an important part of this habitat as it could easily come through to the ground because of a lack of trees. 
Children also explored ideas of events in the wood relating to the sound representing photosynthesis, by reflecting upon the relationship between the sun and plant behaviour. One pair of children, prompted by the remote facilitator to think about the root uptake sound in relation to their moisture readings inferred a particular location in the habitat to be more moist because they had received more root uptake sounds than the photosynthesis sound. This indicated to them the presence of more moisture than sunlight. Here the children are relating quantity of sound to quantity of physical attributes in the wood. This is an interesting inference, and suggests ways in which sound can be modified and used to represent other relevant attributes of the woodland, that would support children's understanding of habitat distributions.

At times the children found it hard to perceive some of the symbolic animal sounds as being 'representational' across a general class of species. For example, sounds that were intended to 'represent' animals-in-general eating at a higher level, were taken to relate to one species, rather than being representative of several. It was almost as if once they had associated a particular animal to a general class behaviour it was hard to imagine it being otherwise. Our original idea was that mapping the sound to the 'general' could increase the exploration or enquiry into the possibilities of a variety of animals inhabiting that particular location. This could also support them in thinking about the most likely organism for the habitat that they are currently in, whether those other animals would be there and if not why not.

As to be expected, the children found it harder to interpret the arbitrary mappings in terms of accurately matching the abstract sounds to the ecological processes. For example, most children found it hard to interpret the arbitrary sound representing photosynthesis (light sabre sound) and decomposition (paper rustling). On the one hand, this implies it is easier for the children to make the connection between a referent that can be imagined sonically (e.g. root uptake) and a sound they are familiar with (slurping) than a hard-to-imagine abstract process and an arbitrary sound. However, abstract processes are inherently difficult to understand. Our primary objective was to draw the children's attention to the underlying processes 
and to get them to think about them in the physical context of the woodland. Indeed, many of the children provided explanations of the process of photosynthesis, using features of the environment, including the sun and leaves, to do this.

Rather than viewing the use of arbritary mappings as problematic, because they are difficult to interpret, we argue they can be useful at provoking other kinds of reflection. In particular, the use of arbitrary mappings can stimulate children to be creative in their explanations, and promote multiple interpretations (13).

\section{Conclusions}

Designing a device to deliver contextually relevant information in the form of sound provided benefits over delivering sound pervasively within the environment. Providing a mixture of pervasive delivery with direct control to access the sounds was successful in overcoming the problem of children missing sounds altogether due to noise. Moreover, collecting and listening to sounds using the Ambient Horn enhanced the children's ongoing activity. In particular, the design allowed the children to access sounds when they were ready to listen to them rather than requiring their immediate attention to hear them, as was the case in the previous study, when the sounds were emitted via wireless speakers. The Ambient Horn also provided a novel way of thinking about ecological processes through a combination of arbitrary and symbolic mappings. The two types of mappings provoked different kinds reflection and interpretation. Symbolic sounds facilitated reflection about invisible organisms or processes and interpretation in relation to the environment. Although the arbitrary sounds were more difficult to interpret and imagine, they, nonetheless, promoted children to think about the underlying processes. 


\section{Acknowledgements.}

This work has been carried out through the EPSRC-funded EQUATOR Interdisciplinary Research Collaboration EPSRC GR/N15986/01 (www.equator.ac.uk). We would particularly like to thank our partners from Sussex (especially Rowanne Fleck), Southampton, Bristol and Nottingham, and participating teachers and pupils from Varndean School, Brighton. Further support was provided by Hewlett Packard's Art and Science programme

\section{References}

1. Ackerman, E. (1996) Perspective-Taking and Object Construction: Two Keys to Learning. In (eds) Y. Kafai and M. Resnick Constructionism in Practice: Designing, Thinking and Learning in a Digital World. Lawrence Erlbaum, NJ.

2. Beaudouin-Lafon, M. and Gaver, W. (1994) ENO: Synthesizing Structured Sound Spaces. Seventh Annual Symposium on User Interface Software and Technology ACM

3. Blattner, M., Sumikawa, D. and Greenberg, R. (1989) Earcons and icons: Their structure and common design principles. Human Computer Interaction 4, 1, 11-44.

4. Brewster, S.A. (1994) Providing a structured method for integrating non-speech audio into human-computer interfaces. PhD Thesis, University of York, UK, 1994.

5. Brewster, S.A., Wright, P.C. and Edwards, A.D.N. (1993) An evaluation of earcons for use in auditory humancomputer interfaces. In Proceedings of InterCHI'93 (Amsterdam) ACM Press, Addison-Wesley, 1993, pp. 222227.

6. Childs, E. (2001) The Sonification of Numerical Fluid Flow Simulations. Proceedings of the 2001 International Conference on Auditory Display, Finland 2001.

7. Drascic, D. and Milgram, P. (1996) Perceptual issues in augmented reality. SPIE:Stereoscopic Displays and Virtual Reality Systems III, Volume 2653, Editors: Mark T. Bolas, Scott S. Fisher, John O. Merritt San Jose, California, USA, January - February 1996, pp 123-134

8. Evard, R. (1993) Collaborative networked communication: MUDs as System tools. Proceedomgs of Seventh Systems Administration Conference (LISA VII), (1993) 1-8.

9. Forrester, D. and Jantzie, N. (2000) http://www.ucalgary.ca/ gnjantzi/learning_theories.htm

10. Francioni, J.M., Allbright, L., and Jackson, J.A. (1992) Debugging parallel programs using sound. SIG-PLAN Notices, 26(12), 68-75.

11. Garcia-Ruiz, M.A. (2001). Using non-speech sounds to convey molecular properties in a virtual environment International Conference of New Technologies in Science Education (CINTEC), Aveiro, Portugal, 4-6 July 2001.

12. Gaver, W. (1986) Auditory icons: Using sound in computer interfaces, Human-Computer Interaction, 2, 1986, 167-177 
13. Gaver, W., Beaver, J., and Benford, S. (2003). Ambiguity as a resource for design. Proceedings of CHI2003, Ft. Lauderdale. New York: ACM Press.

14. Hoyles, C. and Noss, R. (1999) Playing with (and without) words. Proceedings of the seventh European Logo Conference Eurologo '99 Sofia, Bulgaria, 18-29.

15. Ishii, H., and Ullmer, B. (1997). "Tangible bits: Towards seamless interfaces between people, bits and atoms." Proceedings of CHI'97. ACM Press. 234-241.

16. Kaper, H. G., Tipei S, Wiebel, E. (1999) Data sonification and sound visualization, Computing in Science and Engineering, 1(4), 48-58.

17. Kilander, F. and Lönnqvist, P. (2002) A Whisper in the Woods - An Ambient Soundscape for Peripheral Awareness of Remote Processes. Proceedings of the 2002 International Conference on Auditory Display, Japan 2002.

18. McGookin, D. and Brewster, S.A. An Investigation into the Identification of Concurrently Presented Earcons. In Proceedings of ICAD 2003 (Boston, MA). ICAD.

19. Price, S., Rogers, Y., Scaife, M., Stanton, D. \& Neale, H. (2003a) 'Using 'tangibles' to promote novel forms of playful learning'. Interacting with Computers, 15/2, May 2003, pp 169-185.

20. Price, S., Rogers, Y., Stanton, D., and Smith, H. (2003b) A New Conceptual Framework for CSCL: Supporting Diverse Forms of Reflection through Multiple Interactions. In (eds) B. Wasson, S. Ludvigsen, U. Hoppe, Designing for Change in Networked Learning Environments. Proceedings of the International Conference on Computer Supported Collaborative Learning 2003.

21. Resnick, M. (2003). Thinking Like a Tree (and Other Forms of Ecological Thinking) .International Journal of Computers for Mathematical Learning, vol. 8, no. 1, pp. 43-62.

22. Rogers, Y. (1989) Icons at the interface: their usefulness. Interacting with Computers , 1, 105-118

23. Rogers, Y., Scaife, M., Harris, E., Phelps, T., Price, S., Smith, H., Muller, H., Randell, C., Moss, A., Taylor, I., Stanton, D., O'Malley, C., Corke, G. \& Gabrielli, S. (2002a) Things aren't what they seem to be: innovation through technology inspiration. Proceedings of DIS2002, London, 25-28 June, 373-377

24. Rogers, Y., Price, S., Harris, E., Phelps, T., Underwood, M., Wilde, D. \& Smith, H. (2002b) Learning through digitally-augmented learning experiences: Reflections on the Ambient Wood project. (Equator technical report) 25Somers, E. (2000) Abstract Sound Objects to Expand the Vocabulary of Sound Design for Visual and Theatrical Media. Proceedings of the International Conference on Auditory Display. Georgia USA

26. Wilde, D. (2003) The Periscope: Supporting a Computer Enhances Field Trip for Children. First International Conference on Appliance Design, Bristol May 2003 